\title{
Back to the Hospital for Chronic Care: a Hybrid Generalist Model
}

\author{
Jennifer Sumner, $P h D^{1,2,3}$ (c) and Yee Wei Lim, $P h D^{1,2}$
}

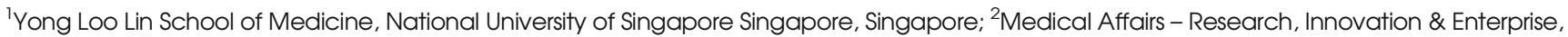
Alexandra Hospital, National University Health System 378 Alexandra Road, Singapore, Singapore; ${ }^{3}$ Department of Health Sciences, University of York, York, UK.

\begin{abstract}
Singapore, like many countries, is attempting to meet the growing healthcare needs of an ageing population with a high burden of chronic diseases. Despite efforts to integrate and increase healthcare capacity, longstanding challenges remain difficult to overcome. Recently, policymakers have considered a new approach to building chronic and eldercare capacity-the Integrated General Hospital (IGH). The development of the IGH model is motivated by a combination of factors: the limit to which the primary care system can manage patients with increasingly complex chronic diseases, a longstanding preference of patients for hospital-based specialty services and patients experiencing fragmented care delivery. The IGH model links hospital care teams and community-based care providers, to facilitate the management of patients throughout the care continuum in a single integrated site. It is hoped that this hospital-led model for chronic care can meet patients' needs and preferences and reduce fragmentation of care.
\end{abstract}

J Gen Intern Med 36(3):782-5

DOI: $10.1007 / \mathrm{s} 11606-020-06271-6$

(C) Society of General Internal Medicine 2020

\section{BACKGROUND}

Singapore, like many countries, has a rapidly ageing population with a growing chronic disease burden. ${ }^{1}$ To meet the growing demand for chronic disease-related healthcare services, Singapore has attempted to provide better coordinated and patient-centred services. Recent initiatives include the development of three integrated health clusters, organised to improve the distribution of facilities, capabilities and services across primary, secondary and community levels. ${ }^{2}$ Another example is the primary care network (PCN), established to enhance primary care through resource sharing, the setting-up of chronic disease clinics and a disease registry. ${ }^{3}$ Despite efforts to integrate healthcare services and improve the capacity of primary care to manage an ageing population, longstanding barriers remain.

Primary care in Singapore is largely privatised. As of 2019, 1700 private practices ( $80 \%$ of primary care) and 20

Received May 11, 2020

Accepted September 25, 2020

Published online October 6, 2020 polyclinics (publicly funded primary care clinics) were in operation. ${ }^{4}$ While polyclinics offer subsidised care, they are heavily subscribed. In the hospital setting, the reverse is true; approximately $70 \%$ of hospitals are public institutions that offer subsidised care. ${ }^{5}$ Consequently, patients routinely seek subsidised chronic disease care from specialists based in hospitals. Singaporeans' perceive that hospital specialists provide a better quality of care, a perception also true in South Korea, Hong Kong and Japan. ${ }^{6-12}$ The combination of a favourable perception of specialty care and the lower cost of subsidised public hospital services has led to an imbalance in chronic disease care delivery between hospitals and the primary care system. The lack of a primary care gatekeeper compounds the situation further because patients are free to "shop" for care from different healthcare providers, i.e. to obtain a desired referral to the hospital specialist of choice. Patients could also seek care directly from hospital specialists if they are willing to pay non-subsidised prices.

This is not to say efforts to move chronic disease care away from hospitals to primary care have not been attempted. The Family Medicine Clinic, established in 2013, aimed to develop a shared care model between private general practitioner (GP) groups and a public hospital. ${ }^{8}$ Resources (staff and equipment) and medical records are shared between GPs and hospitals to better manage chronic disease patients. However, despite satisfaction with the Family Medicine Clinic, patients continued to seek hospital-based specialist care. Reasons included concerns around cost and the perception that care was not comprehensive. The aforementioned PCN, which was set up in $2017^{3}$ to enhance capacity in primary care, has also been slow to adopt — only ten networks were approved in 2018. More recently (in 2019), the Community Health Assist Scheme (CHAS), originally launched in 2012 to facilitate lowerincome household access to subsidised care at private GP clinics, expanded its eligibility to include high-income households. ${ }^{13}$ Since 2012, CHAS has undergone significant enhancements such as removal of age restrictions, increase in coverage for up to 19 chronic conditions and increases in the monthly household income cap and additional cover for common (non-chronic) illnesses. ${ }^{13,14}$ It is hoped CHAS can help to incentivise patients to seek chronic disease care from primary care providers instead of using hospital-based specialists. 
Primary care development and innovation, albeit important and needed, may not be enough to meet the increasing burden and complexity of chronic disease care. We describe a new healthcare model in Singapore, which attempts to further integrate care, build on patients' predilection for hospitalbased care and encourage patients to move away from seeking care from several specialists to receiving care from one care team in one institution.

\section{The Integrated General Hospital Care Model}

The Integrated General Hospital or IGH was launched in 2018 (at Alexandra Hospital), a generalist-led, hospital-based care model, which bridges between hospital specialists and community-based care (Table 1 and Fig. 1). IGH provides medical, surgical and allied health services to the general population. The IGH model adopts a form of care integration that revolves round a generalist physician (usually internal medicine trained) and a team of nurses and allied health providers. Chronic disease patients attending IGH are given the option to replace care by multiple individual specialists with a single generalist-led care team at Alexandra Hospital. This "one patient, one care team" approach is supported by five key inpatient and outpatient programmes: 'Well' (focuses on disease prevention and health promotion), 'FAST' (combines acute, sub-acute and rehabilitative care for inpatients), 'Chronic' (an outpatient clinic focuses on chronic disease management), 'Healthy Ageing' (focuses on patients 65 years and above who require care by a geriatric team) and 'Palliative'. These programmes facilitate the management of patients throughout the care continuum, so as to improve care coordination and reduce fragmentation of care. It is also hoped that integrating chronic disease care within the hospital may be more acceptable to patients who prefer institution-based care.

Table 1 Key Features of the IGH Care Model

\begin{tabular}{|c|c|}
\hline $\begin{array}{l}\text { IGH care model key } \\
\text { features }\end{array}$ & Details \\
\hline $\begin{array}{l}\text { 1. Generalist-led multi- } \\
\text { disciplinary team }\end{array}$ & $\begin{array}{l}\text { - Multi-disciplinary care team led by an } \\
\text { internal medicine physician or specialists } \\
\text { acting as a 'generalists' } \\
\text { - Nurse clinician-led care for lower acuity } \\
\text { patients }\end{array}$ \\
\hline 2. Care consolidation & $\begin{array}{l}\text { - Screening of suitable candidates i.e. multi- } \\
\text { morbid, currently receiving care from } \\
\text { multiple specialists } \\
\text { - Identification of a principal physician } \\
\text { - Discussion with the patient to consolidate } \\
\text { chronic outpatient care at Alexandra } \\
\text { Hospital under a principal 'generalist' } \\
\text { physician }\end{array}$ \\
\hline $\begin{array}{l}\text { 3. One patient, one bed, } \\
\text { one team }\end{array}$ & $\begin{array}{l}\text { - Patients are admitted and managed on one } \\
\text { site, by one team, for the entire care } \\
\text { continuum }\end{array}$ \\
\hline 4. Community outreach & $\begin{array}{l}\text { - Link up with community social services } \\
\text { - Two-way interactions between hospital } \\
\text { and primary care } \\
\text { - 'CareHub'-provides transition care from } \\
\text { hospital to home and address other } \\
\text { home-based health and social needs } \\
\text { - Hospital pharmacist home visits }\end{array}$ \\
\hline
\end{tabular}

IGH, The Integrated Generalist-led Hospital
Outside of the hospital, IGH care teams attempt to link up with community-based services, such as primary care and social care services, to co-manage chronic disease patients - particularly the more complex cases. Similar to the Family Medicine Clinics, local GPs can access IGH's resources such as hospital-based diagnostic services including spirometry and diabetic retinopathy screening without needing to refer patients to hospital specialists. Polyclinics can admit patients to IGH wards without going through the hospital's urgent care clinic for a set of pre-determined medical conditions. IGH's CareHub nurses visit patients who have more frequent admissions to assess their home environment and provide education and support.

In contrast to the IGH model, usual care consists of acute and sub-acute care in separate institutions (Fig. 1). When patients require rehabilitative care following acute care episodes, patients need to be transferred to another hospital. Care across multiple sites can lead to transfer delays and suboptimal information sharing between sites. The role of nurses is also limited in hospital-based usual care. IGH uses nurse-led care for low acuity patients while physician contact is optimised for higher acuity patients. Usual outpatient care of chronic disease patients is typically managed by multiple hospital-based specialists, leading to a lack of care coordination. IGH attempts to overcome this by consolidating all outpatient chronic care under a single principal physician who is supported by specialists.

\section{Challenges in Implementation}

The first year of operation for IGH has not been without challenges. Current internal general medicine physician numbers are limited. IGH inpatient and outpatient medical care teams are therefore led by generalists as well as specialists. Some specialists who have not routinely practiced in a generalist manner have shown hesitancy and scepticism to the new arrangement. Measures have been put in place to empower specialists to practice generalist medicine; these include structured educational sessions (with case discussions), easy access for specialists to consult colleagues in unfamiliar areas of medicine and joint management of patients with complex problems. The other concern among specialists is that practicing generalist medicine at IGH takes away time to develop and practice in their field of expertise. From a specialist career development perspective, this could be seen as unattractive.

From the patient's perspective, changing established healthcare seeking behaviour is not straightforward. Many patients with multiple chronic diseases have become accustomed to seeking care from multiple physicians, sometimes from different institutions. They have formed relationships with their doctors. When patients are asked to consolidate their care to one generalist, they could be reluctant to abandon long-term relationships with their current physicians. Patients could also be unwilling to consolidate their care at Alexandra Hospital if they do not live within the immediate area. While 


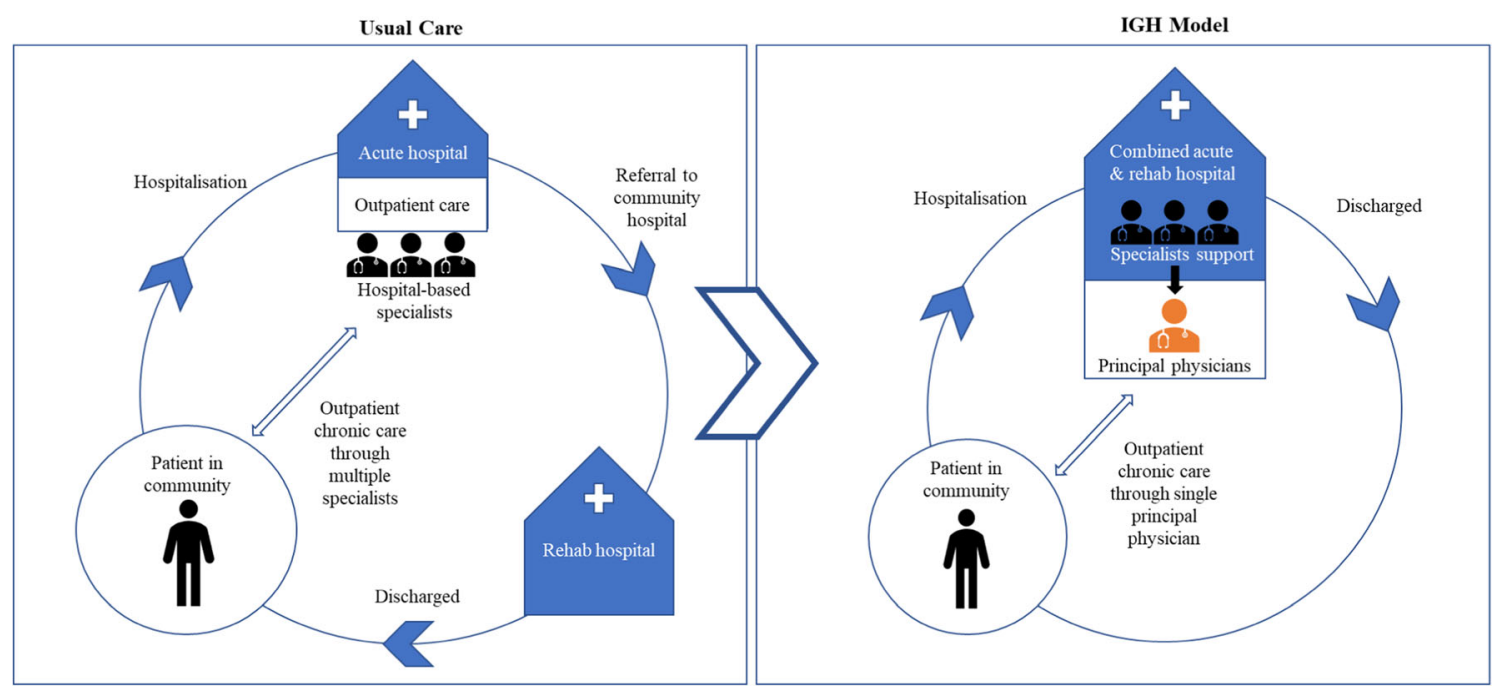

Figure 1 Typical patient pathway for acute, sub-acute and specialist care versus IGH model.

many patients acknowledge the benefit of integrating care, considering the trade-offs between their existing care relationships versus adoption of a new care team, the choice is not always easy. Nevertheless, IGH care teams are making efforts to educate patients about the advantages of care consolidation and to reassure them that consolidating care does not mean reduced access to specialist care.

At the systems level, there are challenges to adopting the IGH care model. Hospital-based services are more costly than community-based services. In the long run, hospital-led chronic disease care models may be too costly to sustain even if patients prefer it. China, which almost exclusively used a hospital-based care approach, is now re-organising hospitals to focus on acute care while simultaneously developing the primary care sector. The drivers for China's reorganisation were cost reduction and quality improvement. ${ }^{15}$ Similarly, healthcare reforms in Japan are attempting to move primary care needs, traditionally served by costly specialist-led clinics or hospital-based clinics, towards more cost-effective primary care practices, led by GPs. ${ }^{12}$ This is not to say hospital-based care is not needed. Patients with more complex care needs might be more cost-effectively managed by hospital-based generalists (supported by specialists). But for less complex cases, patients may be better served in the primary care setting. The challenge for IGH is thus to develop a shared care model for both complex and non-complex patients with the primary care sector, which is also welcomed by patients and financially sustainable. This may require expanding IGH nurses' and allied health providers' roles in chronic disease care, increasing adoption of technological solutions, such as tele-health services, and to plant IGH-like resources in the community, such as shared clinical sessions between IGH care teams and its primary care partners.

A piecemeal approach to support chronic care capacity development in the private primary care sector is a reason IGH is relevant today. Only within the last seven years have initiatives emerged (e.g. FMC, PCN and extension of CHAS) to support private primary care providers to better manage patients with chronic diseases. It will take time for these initiatives to mature and involve more private providers. However, IGH's development may convey a perception that investment in primary care is not top priority and alienate the sector. The undervaluing of primary healthcare is unfortunately not unusual. ${ }^{16}$ Despite evidence of better health outcomes, and reduced cost of care, efforts to build a stronger primary care system are not consistent. ${ }^{16,17}$

It seems counterintuitive to move patients to the hospital for chronic disease care and may even appear to perpetuate a model of hospital-led rather than community-led care. Perhaps the IGH model should be viewed as a pragmatic supplemental approach as the primary care system continues to grow. In the future, IGH could shift its focus to more complex and highneed patients, while working closely with primary care partners to ensure continuity of care inside and outside of the hospital for all patients. There are plans to replicate IGH model across Singapore. Some aspects of IGH, such as the combination of acute and rehabilitative care on a single site, could be adopted to reduce care fragmentation and reduce overall length of stay. Other aspects, such as care consolidation, and the use of teleheath will require further evaluation before they are implemented more widely.

\section{CONCLUSION}

The care burden of an ageing population will continue to grow in the coming years. IGH has the potential to complement existing efforts to develop chronic disease care capacity. By consolidating acute and sub-acute care in one site and integrating care through a generalist-led care team approach, IGH could be a valuable partner for primary care and community providers. IGH does not pretend to replace primary care as the medical home for patients with chronic care needs. Rather its future lies in optimising shared care with primary 
services - particularly for complex cases. Advances in infrastructure are needed to support effective shared care. Moving forward, the challenge is to explore IGH's scalability and sustainability, its ability to grow in tandem and integrate with the rest of the healthcare system.

Corresponding Author: Jennifer Sumner, PhD; Department of Health Sciences, University of York, York, UK (e-mail: jenny. sumner@york.ac.uk).

\section{Compliance with Ethical Standards:}

Conflict of Interest: The authors declare that they do not have a conflict of interest.

\section{REFERENCES}

1. Wang $\mathbf{H}$, Naghavi $\mathbf{M}$, Allen $\mathbf{C}$, et al. Global, regional, and national life expectancy, all-cause mortality, and cause-specific mortality for 249 causes of death, 1980-2015: a systematic analysis for the Global Burden of Disease Study 2015. The Lancet. 2016;388(10053): 1459-1544.

2. Ministry of Health. Reorganisation of healthcare system into three integrated clusters to better meet future healthcare needs. 2018 https://www.moh.gov.sg/news-highlights/details/reorganisation-ofhealthcare-system-into-three-integrated-clusters-to-better-meet-futurehealthcare-needs. Accessed 11 March 2020.

3. Ministry of Health. Primary Care Network. 2018; https://www.moh.gov. sg/our-healthcare-system/healthcare-services-and-facilities/primarycare-networks. Accessed 11 March 2020.

4. Ministry of Health. Primary healthcare services. 2019; https://www.moh. gov.sg/home/our-healthcare-system/healthcare-services-and-facilities/ primary-healthcare-services. Accessed 17 July 2020.

5. Ministry of Health. Health facilities. 2019; https://www.moh.gov.sg/ resources-statistics/singapore-health-facts/health-facilities. Accessed 24 July 2020.

6. van Weel C, Kassai R. Expanding primary care in South and East Asia British Medical Journal. 2017;356. doi:https://doi.org/10.1136/bmj. j634
7. Montegut AJ, Cartwright CA, Schirmer JM, Cummings S. An international consultation: the development of family medicine in Vietnam. Family medicine. 2004;36(5):352-356.

8. $\operatorname{Lim} \mathbf{Y W}$, Ling $\mathbf{J}, \operatorname{Lim} \mathbf{Z}$, Chia A. Family Medicine Clinic: a case study of a hospital-family medicine practice redesign to improve chronic disease care in the community in Singapore. Family practice. 2018;35(5):612 618

9. Ock. M, Kim. J, Jo. M, Lee. H, Kim. HJ, Lee. JY. Perceptions of primary care in Korea: a comparison of patient and physician focus group discussions. BMC Family Practice. 2014;15. https://doi.org/10.1186/ s12875-014-0178-5.

10. Kim. AM, Cho. S, Kim. HJ, et al. Primary Care Patients' Preference for Hospitals over Clinics in Korea. International Journal of Environmental Research and Public Health. 2018;15. https://doi.org/10.3390/ ijerph 15061119 .

11. Wong. SYS, Kung. K, Griffiths. SM, et al. Comparison of primary care experiences among adults in general outpatient clinics and private general practice clinics in Hong Kong. BMC Public Health. 2010;10. https://doi.org/10.1186/1471-2458-10-397.

12. Kato. D, Ryu. H, Matsumoto. T, et al. Building primary care in Japan: Literature review. Journal of General and Family Medicine. 2019;20:170179

13. Ministry of Health. Community health assist scheme. 2019; https:// www.moh.gov.sg/news-highlights/details/speech-by-mr-gan-kim-yongminister-for-health-at-the-community-health-assist-scheme-(chas)-carnival-27-july-2019. Accessed 17 July 2020.

14. Agency for Integrated Care. Community health assist scheme (CHAS) factsheet. 2017; https://partners.aic.sg/sites/aicassets/AssetGallery/Factsheets/ Factsheet\%20for\%20CHAS\%20Family\%20Carnival\%202017_FINAL.pdf. Accessed 29 July 2020

15. World Bank Group, World Health Organization, Ministry of Finance, National Health and Family Planning Commission, Ministry of Human Resources and Social Security. Deepening health reform in China: Building High-Quality And Value-Based Service Delivery. 2016

16. Rao. M, Pilot. E. The missing link - the role of primary care in global health. Global Health Action. 2014;7. doi:https://doi.org/10.3402/gha. v7.23693.

17. World Health Organization. Building the economic case for primary health care: a scoping review. Geneva: WHO;2018.

Publisher's Note: Springer Nature remains neutral with regard to jurisdictional claims in published maps and institutional affiliations. 